\title{
LA EMERGENCIA Y EXPANSIÓN DEL COOPERATIVISMO EN BRASIL
}

\author{
Daniel Francisco Nagao Menezes \\ Profesor del Programa de Posgrado en Derecho Político y Económico \\ Universidade Presbiteriana Mackenzie (Brasil) \\ Miembro de CIRIEC-Brasil \\ https://orcid.org/0000-0001-9151-5699
}

\section{RESUMEN}

Este artículo está dedicado a analizar el surgimiento y expansión del movimiento cooperativo en Brasil. El artículo es relevante al observar qué arreglos adaptativos ocurrieron en Brasil al utilizar conceptos europeos en la construcción del cooperativismo brasileño. También demuestra la existencia de una relación muy específica con el Estado, diferente a otros países, oscilantes períodos de indiferencia del Estado en relación a la cooperativa, alternando con períodos de combate y otros de promoción del cooperativismo. El objetivo es permitir la comprensión de la situación actual del cooperativismo en Brasil, dividida entre grandes empresas económicas que se desvían de los principios originales del cooperativismo y; un cooperativismo popular, destinado a sobrevivir a las adversidades económicas. El texto es descriptivo, presenta un momento histórico brasileño, no busca responder una pregunta de investigación específica.

PALABRAS CLAVE: Cooperativas, relación con el Estado, Historia de las Cooperativas, Brasil.

CLAVES ECONLIT / ECONLIT DESCRIPTORS: J54, N00, P32.

Cómo citar este artículo/How to cite this article: NAGAO MENEZES, D.F.: "La emergencia y expansión del cooperativismo en Brasil", CIRIEC-España, Revista Jurídica de Economía Social y Cooperativa, $\mathrm{n}^{\circ}$ 39, 2021, pp. 285-303. DOI: 10.7203/CIRIEC-JUR.39.21941 
(pp. 285-303)

\section{THE EMERGENCE AND EXPANSION OF COOPERATIVISM IN BRAZIL}

\section{EXPANDED ABSTRACT}

Even considering the specific characteristics of management and organization of cooperatives, in Brazil the role of the State in the expansion of agricultural cooperatives was of fundamental importance. A set of measures allowed the formation of cooperatives aimed at agro-industrial activities due to the modernization of agriculture in the second half of the 20th century.

Latin American countries are characterized by greater dependence on the State for the development of economic activities and implementation of social rights. For this reason, the history of cooperativism in Brazil is the history of the State's treatment of cooperative societies.

Initially, Brazil has a period of indifference by the State in relation to cooperatives, which goes from the end of the 19th century to 1930. During this period, the State did not prohibit and also did not encourage cooperativism as an economic strategy. Groups wishing to found a cooperative should apply for authorization from the state.

The second period runs from 1930 to 1950 , when cooperatives were prohibited by the state as they were mistaken for a communist organization. Only a few cooperatives linked to unions loyal to the government were allowed to function. At this time, Brazil lived the dictatorship of Getúlio Vargas and, it is interesting to note that Mexico (Cardenas) and Argentina (Perón), created a relationship with cooperativism very similar to that which existed in Brazil.

The third period starts in the 60 s and is in effect until today. From that moment on, the State adopted cooperativism as a strategy for the development of some strategic sectors, such as agriculture, pushing cooperativism away from its original principles..

During the $60 \mathrm{~s}$, the institutional representation of Brazilian cooperativism was divided into two: the Brazilian Association of Cooperatives (ABCOOP) and the National Union of Cooperatives (Unasco), and it was only in 1969 that the Organization of Brazilian Cooperatives (OCB) was created, unifying the two entities. $O C B$ assumed the role of representing and centralizing measures related to Brazilian cooperatives but controlled by the State. OCB thus became accredited to provide legal support for a new format for business cooperativism in Brazil.

Therefore, since that time, in Brazil, two large groups can be defined in relation to the cooperative movement. The first represents traditional and older cooperatives, formed in the 
mid-twentieth century and which the Organization of Brazilian Cooperatives - OCB reorganized with an economic focus and establishment in the market, and, on the other hand, are the so-called social cooperatives, which are part of Solidarity Economy, which until 2016 had a Special Secretariat in the federal government, linked to the Ministry of Labor, therefore with a social focus.

In this text, aspects inherent to the so-called corporate/multi-cooperative cooperativism will be discussed, therefore, the one with an economic-mercantile focus. This cooperativism was consolidated in Brazil, as already mentioned, after the emergence of the OCB system, which, since the 1970s, has promoted the sector through union representation, political presence, information, monitoring and social promotion.

In business/multi-cooperative cooperatives, contradictions express dialectical pairs (and not just dualisms) and these pairs must be critically analyzed, such as the territorialization of cooperatives in the perspective of integrating the political, economic and cultural dimensions. In this methodological path, therefore, followed by the dialectical method, subject and object are intrinsic: the subject is constructed/transformed, building/transforming, and, at the same time, the object, which, once under construction, or already built, being the fruit of human work influences the subject's actions.

The development model in Brazil is agro-export, marked by exports as an exogenous variable, which generated an important portion of national income and which supported imports that significantly supplied part of the domestic demand. Cooperative norms are contained in the constitutional context, in the Civil Code and in a special legislation, which is the Law of Cooperative Societies. First cooperative societies were formed and then law regulated them. Now, the Brazilian Federal Constitutions and their influence on cooperativism deserve to be highlighted and, then, the issues pertinent to the General Law of Cooperativism, that is, Federal Law 5.764/1971, will be discussed.

The pursuit of public policies in the course of the twentieth century was aimed at implementing the modernization of agriculture in small and medium-sized production units, which is why the role of agricultural cooperatives was extremely important, aiming to optimize and maximize this path. Then industrialization expanded rapidly and started to demand the restructuring of the field, since it is a source of raw material. It was necessary to prepare the field to produce for industry and, at the same time, for the field to also receive industrialized products, such as pesticides, fertilizers, modified seeds and machines.

The rules contained in the current Federal Constitution and in the specific legislation on cooperatives were being shaped for the development of multi-cooperatives. Therefore, in 
this long way from the Republican Constitution of 1891 until the Citizen Constitution of 1988 , the main guidelines were corroborating a strengthening of the preference for business cooperativism.

In this context, cooperatives become the mediators between public policies and small and medium productive units for the introduction of capitalism in the countryside. These are profound transformations in cooperative organizations, transformations that are based on the re-signification of the old cooperativism brought from Europe and that currently lives highly dialectical relations, strongly approaching the business logic and, thus, it moves away from the cooperative principles formulated by the cooperative members from Rochdale, England. This continuous process of industrialization of rural areas brought about the fragmentation of transformations in the relations of production in agriculture and, consequently, redefined the socioeconomic and political structure in the Brazilian field.

In the past, cooperativism emerged to defend workers excluded from the labor market because of the Industrial Revolution. Since it appeared, therefore, it has been undergoing transformations and becoming a hybrid, to meet the demands of workers and capital, and precarious its cooperative principles. When cooperativism assumes the needs of capital and approaches corporate values, it begins to experience contradictions within the system, experiencing the dilemma between cooperative principles, cooperative members, the market and competitiveness.

Although the modernization of agriculture began in the 1950s, it was from the 1970s that transformations began to be felt at the national level, with the implementation of State programs aimed at improving and expanding production areas. So-called CAIs (Agroindustrial Complexes) were instituted in Brazil, which increasingly demanded changes in the way of producing in the field. With the help of the government, the implementation of the Agro-Industrial Complexes that occurred in the 1970s promoted the intersectoral integration of three basic elements: the industries that produce for agriculture, modern agriculture and the agro-industries. And, together with this new productive structure, cooperatives were also being reorganized, which became multi-cooperative calls and started to operate in networks to meet the new requirements. Industrial capital started to command the rural economy, shaping cooperatives according to their interests. Then there was an intense process of agro-industrialization of cooperatives in the 1980s, especially in the southern region of Brazil. Agricultural cooperatives have become agro-industrial cooperatives, serving the rural in all phases of the production process and integrating productive units with financial capital, always directed by the State through the implementation of specific public policies. 
Brazil is experiencing a historic opportunity to inaugurate a fourth stage in the history of Brazilian cooperativism, starting with agricultural cooperatives. The proposal for this fourth stage is to couple cooperativism to a strategy to overcome underdevelopment, as advocated by organizations such as ECLAC and authors such as Celso Furtado and Raul Prebisch.

This will result in the redirection of cooperative production to the domestic market, moving away from the dependence that large agricultural cooperatives have on the foreign market, especially China and the United States. This will reduce the dependence of large Brazilian agricultural cooperatives on the international market and, on the other hand, will increase the power of small businesses, returning to the old cooperative principles of Rochdale.

KEYWORDS: Cooperatives, Relationship with the State, History of Cooperatives, Brazil. 
(pp. 285-303)

\section{SUMARIO}

1. Formación del cooperativismo en Brasil. 2. La creación de la ley actual. 2.1. La Organización de Cooperativas Brasileñas - OCB. 2.2. Cooperativas agrícolas en Brasil. Referencias.

\section{Formación del cooperativismo en Brasil}

En Brasil, las primeras experiencias colectivistas se estructuran sobre el modelo integral de cooperación. El primer proyecto se remonta a 1610 con la fundación de las primeras misiones de los sacerdotes jesuitas en Brasil. El modelo de los jesuitas se basó en el colectivismo, fundado en el trabajo colectivo de los miembros, basado en el bienestar de las familias, superponiendo el interés económico de la producción. La acción de los jesuitas se basó en la convicción de los principios del amor cristiano (amor al prójimo) y del principio de ayuda mutua, ya existentes entre los indígenas brasileńos y otros pueblos primitivos de América.

Hay pocos registros históricos de formas de cooperación y otras experiencias colectivas en el Brasil colonial y durante el Imperio. Se sabe que los pueblos indígenas desarrollaron una organización económica basada en el primitivo modo de producción comunal ${ }^{1}$, estructurada en caza, pesca, recolección de frutos y en agricultura de subsistencia. Incluso hoy, las tribus que aún se resisten organizan su trabajo en tareas colectivas de recolección y producción. Estas relaciones colectivistas fueron mejoradas por los indios guaraníes en las Reducciones de los jesuitas.

Durante el Brasil colonial, las Reducciones representaron la única experiencia de empresas colectivas, basadas en el comunismo primitivo de las organizaciones sociales indígenas, junto con la doctrina católica del comunitarismo como la organización ideal de los primeros cristianos, como se encuentra en los textos bíblicos.

Estas empresas (Reducciones) duraron entre 1610 y 1767, teniendo una amplia extensión en América del Sur (se encuentran en Argentina y Paraguay y, en Brasil,

1. "Uma relação social denomina-se "relação comunitária" quando e na medida em que a atitude na ação social - no caso particular ou em média ou no tipo puro - repousa no sentimento subjetivo dos participantes de pertencer (afetiva ou tradicionalmente) ao mesmo grupo. Uma relação social denomina-se "relação associativa" quando e na medida em que a atitude na ação social repousa num ajuste ou na uniäo de interesses racionalmente motivados (com referência a valores ou fins). A relação associativa, como no caso típico, pode repousar especialmente (mas não unicamente) num acordo racional, por declaração recíproca. Então a ação correspondente, quando é racional, está orientada: a) de maneira racional referente a valores, pela crença no compromisso próprio; b) de maneira racional referente a fins pela expectativa da lealdade da outra parte."(WEBER, 2004, p. 25). 
desde el norte de Paraná hasta Rio Grande do Sul). Debido a la ausencia de registros históricos, el legado de las Reducciones es insignificante.

El movimiento cooperativo, de modelo europeo, se inició en Brasil en $1847^{2}$, por la acción del francés Jean Maurice Faivre, quien fundó, junto a un grupo de europeos, en el interior del Estado de Paraná, la colonia Teresa Cristina. La colonia agrícola sentó las bases del cooperativismo brasileño y, aunque poco reconocida, es la primera empresa colectiva brasileña. Posteriormente, en 1890, se fundó otra empresa, Colônia Cecília, ubicada en Palmeira, estado de Paraná, organizada por Giovanni Rossi, militante anarquista italiano. La colonia Cecília tiene un sesgo ideológico anarquista, recibiendo un fuerte apoyo internacional (ZECCA CASTEL, 2008; MELLO NETO, 1998), siendo una experiencia utópica estructurada en el trabajo colectivo y el amor libertario, con una duración no mayor de cuatro años y con no más de 300 participantes.

Algunos puntos en común en estos proyectos primitivos en Brasil se encuentran como la miseria material casi absoluta y la hostilidad de las comunidades vecinas a los proyectos y del propio Estado, que vieron en estas Colonias una amenaza de implantación del anarquismo en Brasil. Por otro lado, algunos legados y experiencias únicas provienen de estas dos colonias, como la existencia de tierras comunitarias producto de diversos factores que van desde las precarias condiciones del proceso de colonización brasileña hasta las tradiciones culturales, tanto indígenas como de inmigrantes europeos, especialmente anarquistas. Estas condiciones permitieron la existencia de áreas de tierra como propiedad comunal, sin apropiación privada, que fueron utilizadas por todas las familias ${ }^{3}$.

Un punto común de las empresas originarias del sur de Brasil es la naturaleza de un movimiento social, de carácter económico, ya que apuntaba a una mejor organización productiva y financiamiento de actividades, especialmente para los pequeños productores rurales y; también, cultural, ya que la empresa servía como lugar de reproducción y mantenimiento de las tradiciones de los inmigrantes que la integra-

2. Antes de eso, hubo un experimento fallido en 1842, en el que el médico francés Benoit Mure instigó la creación de un falansterio (dentro del modelo de Fourier) en la región de Sáo Francisco do Sul, Estado de Santa Catarina, y que duró solo cuatro años.

3. Hasta el día de hoy, en el sur de Brasil, existe una forma similar de propiedad colectiva llamada "faxinal". Los faxinais son áreas de bosque y pastos, que se utilizan de manera comunitaria para el pastoreo de ganado y el suministro de madera a los residentes de los alrededores. El sistema de limpieza es "tradicionalmente caracterizado pelo uso coletivo da terra para a criação de animais, sendo denominado 'criadouro comum', pelo fato dos animais serem criados em regime de compáscuo. O faxinal é dividido em terras de plantar, que são destinadas ao cultivo agricola, sendo delimitadas por uma cerca construida coletivamente; e as terras de criar, destinadas à criação de animais de várias espécies" (SOCHODOLAK \& MANEIRA, 2011, p. 3). A pesar del uso comunal de la tierra, la propiedad del ganado es individual. 
ban (ICAZA \& ASSEBURG, 2004, p. 23). Recién en los años 60 del siglo pasado las empresas abandonaron estas características y asumieron una matriz económica empresarial.

Cuarenta años después de los emprendimientos originales (Colônias), en 1887, se fundaron las primeras cooperativas en Brasil, como la Cooperativa de Consumidores de Empleados Companhia Paulista, en la ciudad de Campinas, Estado de São Paulo, (aunque no registrada oficialmente).

La primera cooperativa de crédito se remonta a 1902, organizada por enólogos de Nova Petrópolis, Estado do Rio Grande do Sul, bajo la dirección del sacerdote jesuita suizo Théodor Amstadt. Esta cooperativa fue concebida como un fondo de crédito rural en la línea de Raiffeisen Caixas, entonces en pleno desarrollo en Europa (diseñado por Friedrich Raiffeisen, alcalde de un pequeño pueblo de Alemania). Esta cooperativa, Cooperural, todavía existe hoy.

En 1908, se creó la Cooperativa Internacional da Lapa, formada por empleados británicos del Ferrocarril de São Paulo. Poco después, en 1911, se creó la Cooperativa Companhia Paulista. En 1913, en Santa María/RS, se crea la Cooperativa de Consumo de Empleados Ferroviarios (Coopfer), pionera en múltiples iniciativas sociales, convirtiéndose en la cooperativa de consumo más grande de Sudamérica (VEIGA \& FONSECA, 2001, p. 21).

Cabe señalar que las primeras cooperativas en Brasil nacieron de representantes de una clase económica ${ }^{4}$ (especialmente los ferroviarios que representaban una "elite" laboral en ese momento), todos vinculados al consumo, apoyados por las grandes empresas (empresas de transporte ferroviario). Actualmente no existe un sesgo puramente popular en las primeras cooperativas brasileñas, sino un fuerte sesgo empresarial.

De los diversos intentos en el sur del país, la que de hecho puede considerarse la primera cooperativa registrada oficialmente en Brasil es la Associação Cooperativa dos Empregados da Companhia Telefônica, de Limeira/SP, con fecha de 1891. El período de consolidación del cooperativismo en el país coincide con la llegada de inmigrantes alemanes, italianos y japoneses, quienes se asentaron en el sur y sureste

4. "Apesar de algumas conquistas dignas de aplausos, o nosso movimento cooperativo apresenta graves defeitos. Ele não é, geralmente, um movimento de origem popular, à semelhança do que se deu em muitos outros países. As cooperativas de consumo, sobretudo as antigas, foram organizadas, muitas vezes, por patróes desejosos de suavizar a situação econômica dos seus empregados, mas sem interesse em um movimento popular de solidariedade. Dai generalizaram-se as cooperativas de classe, que perderam a rica inspiração humana do cooperativismo de Rochdale. Por outra parte, as cooperativas agricolas, bem como as de crédito, foram promovidas, em grande parte, pelo Ministério da Agricultura, com o fim de fomentar a produção. Demais, elas são constituidas, ainda em boa parte, de fazendeiros e usineiros ricos, dominados frequentemente por uma mentalidade capitalista" (MAURER JÚNIOR, 1966, p. 67). 
del país, recordando que en Limeira tuvo lugar la primera experiencia con el trabajo de inmigrantes de Brasil.

En el campo legal, la gran innovación ocurrió solo en el gobierno de Vargas, a través del Decreto No. 22.239, del 19 de diciembre de 1932, el primer documento que regula el cooperativismo en Brasil. Antes de eso, solo existían el Decreto 979 de 1903 y el Decreto 1.637 de 1907, que abordó superficialmente a las cooperativas, estableciendo que las cooperativas adoptan la forma jurídica de una sociedad mercantil existente, como sociedad anónima o sociedad limitada (BULGARELLI, 1995, p. 270).

El Decreto de 1932 organizó principalmente cooperativas de crédito, teniendo como modelo las cooperativas de crédito modelo Raiffeisen, común en el sur de Brasil, cuyas principales características eran: a) la no remuneración de los directores; b) transferencias de sobras a fondos; c) área de acción reducida; d) responsabilidad ilimitada y solidaria de los socios; e) mantenimiento del fondo de reserva y; f) integración entre cooperativas.

Esta situación es resultado del crecimiento del cooperativismo en el país, ocurrido a partir de 1932, motivado por dos puntos básicos: a) el estímulo estatal al cooperativismo al identificarlo como un instrumento de reestructuración de las actividades agrícolas y; b) promulgación de la ley básica del cooperativismo brasileño, de 1932, con el Decreto 22.239/32, que crea la definición legal del cooperativismo y otras formas de asociación (PINHO, 1996, p. 99).

Por un lado, el Decreto 22.239/32 organiza institucionalmente el cooperativismo en Brasil, facilitando el registro de sociedades cooperativas, sin embargo, por otro lado, al no establecer mecanismos de control e inspección, permitió el uso de cooperativas para diversas fraudes, un estigma que acompaña el cooperativismo hasta nuestros días.

Para remediar la omisión del Decreto 22.239/32, muchas otras legislaciones intentaron corregir el problema. Así, en agosto de 1938 se publicó el Decreto Ley 581, creando el Registro Administrativo de Cooperativas con la Dirección de Organización y Defensa de la Producción, posteriormente sustituida por SER - Servicio de Economía Rural, vinculado al Ministerio de Agricultura. El Decreto-ley 6.980 de 19/03/1941 atribuye al Ministerio de Hacienda la competencia de fiscalizar las cooperativas de crédito. En 1945, mediante el Decreto Ley 8401, la supervisión de las cooperativas en general fue transferida al Servicio de Economía Rural, incluidas las cooperativas de crédito.

En 1957, mediante el Decreto 41.872, el poder de inspección de las cooperativas de ahorro y crédito fue compartido entre SER y SUMOC (Superintendencia de Moneda y Crédito). Nueve meses después, este Decreto es derogado por el Decreto 
43.552 y la competencia vuelve a ser exclusiva de la SER, tema que solo se resolvió en 1964 con la Ley 4.595 (Ley de Reforma Bancaria), que atribuye la competencia en materia financiera al Banco Central de Brasil.

Esto crea una gran desorganización en las cooperativas, impidiéndoles organizarse sistemáticamente. Esto pretende ser subsanado por el Decreto Ley 59 de 1966, reglamentado por el Decreto 60.597/67. Sin embargo, la pretensión organizativa del Decreto-Ley, que fue ejercida por la recién creada CNC - Consejo Nacional Cooperativo, generó repulsión de gran parte del cooperativismo brasileño, que criticó el excesivo control y supervisión de la CNC.

La práctica de la CNC, sin embargo, tuvo el efecto contrario a lo que pretendía el Decreto Ley 59/66. La CNC no pudo crear ninguna política dirigida al cooperativismo, no lo organizó como un sistema y no evitó la continuación del fraude, especialmente en las cooperativas de ahorro y crédito, ya que no ejerció su poder de supervisión.

Incluso con buenas iniciativas, casi todas voluntarias, el cooperativismo surgió en el país sin cohesión social y la representación necesaria para componer intereses comunes a nivel nacional y, sin una definición política clara de lo que se pretendía con el movimiento cooperativo, p. la legislación inocua del sector. Recién en los años 50 del siglo pasado surgieron las primeras iniciativas unificadoras del movimiento cooperativo brasileño a través de la creación de UNASCO - Unión Nacional de Asociaciones Cooperativas, que luego se dividiría en ABCOOP - Asociación Brasileña de Cooperativas, debido a diferencias políticas.

La división de representación del movimiento se extiende hasta 1969, cuando el gobierno militar unifica las dos entidades cooperativas brasileñas (UNASCO y ABCOOP) lo que luego daría lugar a la creación de la OCB - Organización de Cooperativas Brasileñas y, de las contrapartes estatales, la OCE - Cooperativa Estatal. Organizaciones. La creación de la OCB se decidió luego de un largo debate entre las cooperativas durante el VI Congreso de Cooperativismo, realizado del 2 al 6 de diciembre de 1969, en Belo Horizonte, Estado de Minas Gerais.

\section{La creación de la ley actual}

Con la Ley $\mathrm{N}^{\circ}$ 5.764, del 16 de diciembre de 1971, se creó legalmente el sistema $\mathrm{OCB}$, con actividades que comenzarán al año siguiente en la sede de la organización en Brasilia, capital de Brasil.

La idea central de la Ley 5.764/71 fue la concentración de cooperativas agrícolas para organizar las exportaciones brasileñas de bienes primarios. Independientemente 
de la caracterización errónea de los ideales cooperativos que fueron infringidos por la ley, el contexto internacional, ya sea con los choques petroleros o el cambio en los patrones de producción (modelo fordista), impidió el avance del cooperativismo en el país y redujo significativamente el patrón socioeconómico nacional, impidiendo la continuidad del proyecto de desarrollo nacional de Getúlio Vargas 5 .

Actualmente, el intento de revitalizar las prácticas cooperativas es parte de un movimiento más amplio para modernizar las actividades productivas en el país y expandir la democracia, lo cual se hace a través de discusiones sobre la economía solidaria y el tercer sector. Estos puntos (economía solidaria y tercer sector) ganan espacio en la agenda de la academia, de los órganos representativos del cooperativismo, el movimiento sindical y el Poder Público.

La perspectiva actual dista mucho de la vivida en la década de los ochenta del siglo pasado, cuando hubo una amplia denuncia contra el cooperativismo brasileño, calificándolo de llevar a cabo una "modernización conservadora" en la que el Estado actuó de manera autoritaria y centralizada, contrario a los principios fundacionales del cooperativismo.

El modelo conservador de modernización favoreció a los medianos y grandes agricultores enfocados en la cultura exportadora, en detrimento de la agricultura de subsistencia desarrollada por la agricultura familiar (SCHNEIDER, 1981, p. 13). El modelo adoptado en Brasil, al igual que en otros países de América Latina, utilizó el cooperativismo como instrumento de control social y político ${ }^{6}$, o incluso como

5. "Esses desafios e dificuldades vieram à tona no rico debate realizado no pós-Segunda Guerra Mundial. Enfatizou-se que faltavam aos paises em desenvolvimento requisitos sem os quais o mecanismo de mercado deixaria de produzir os estímulos ao desenvolvimento. Tratava-se das falhas de mercado, que incluiam falta de infraestrutura de transportes e energia, fraca capacidade empresarial, ausência de um sistema financeiro sólido e baixo grau de qualificaçâo educacional. Faltava, também, capacidade estatal necessária para regular os mercados, estabilizar a moeda e suprir as referidas falhas de mercado. A essas lacunas somavam-se as particularidades do processo de modernização, que se deu de forma dual e desequilibrada, com a coexistência de setores modernos e arcaicos. Esses paises sofriam também os impactos de sua estrutura demográfica, o que acarretou processos de industrialização acompanhados de oferta ilimitada de mão de obra, com impactos negativos sobre a distribuiçäo de renda. Apesar dos constrangimentos, muitos paises se industrializaram e cresceram. A industrialização, no entanto, falhou ao não transformar signifcativamente a estrutura dual dessas sociedades. Os resultados em termos de redução da pobreza e de erradicação da miséria foram muito abaixo do esperado. Esses eventos estimularam a revisão do debate sobre as estratégias de desenvolvimento. Apesar da ênfase acertada na correção das falhas de mercado, constatou-se que havia sido dado um peso excessivo aos aspectos econômicos, acreditando-se que as demais transformaçôes viriam como consequência. Autores, como Amartya Sen (2000), inverteram a ênfase, destacando que segmentos da população, por não possuírem acesso à educação, à saúde e a outros serviços básicos, se tornavam incapazes de beneficiar-se dos estímulos trazidos pelo crescimento. Seria, portanto, necessário atuar diretamente sobre esses fatores, o que incluia, também, abrir maiores oportunidades de participação e fortalecer o papel da mulher dentro do lar." (GUIMARĀES, 2011, p. 318).

6. "Mas como os resultados da pesquisa evidenciaram, o trabalho não é, na maioria dos casos, uma escolha do trabalhador. Há uma absoluta dominância de associaçâo e (ou) cooperativas geradas pela indução da política pública. 
política de gestión de la pobreza (CUNHA \& SANTOS, 2011, p. 23). A diferencia de Europa, donde el cooperativismo nació como una forma de organización proletaria, en Brasil (y en América Latina) el movimiento se estructura como una forma de organizar y promover las élites políticas y agrarias.

\subsection{La Organización de Cooperativas Brasileñas - OCB}

La falta de identidad ideológica entre las representaciones cooperativistas brasileñas y los movimientos populares se hizo explícita con la creación de la OCB en 1969. Concebida para pacificar el conflicto sobre la representación existente y centralizar las diferentes fuerzas del cooperativismo nacional en ese momento en una sola institución. El organismo permite el predominio de los intereses políticos, económicos e ideológicos de los productores rurales y, con el apoyo de los militares, la OCB puede dirigir inversiones sustanciales, especialmente en la agricultura, así como crear privilegios fiscales para sus miembros, especialmente para las cooperativas agrícolas. destinado a la exportación.

Así, Brasil adoptó la posición de Alianza Cooperativa Internacional, y constituye una organización, de carácter jurídico privado, que ostenta, en nombre del Estado, la representación de lo que se llamaría el "sistema cooperativo brasileño".

Con la creación de la $\mathrm{OCB}$, las inversiones estatales se dirigieron a proyectos de creación y fortalecimiento de complejos agroindustriales, vinculados a la política de modernización conservadora del campo que estuvo vigente durante todo el régimen militar. El apoyo estatal a las cooperativas se estructuró en torno a la provisión de crédito subsidiado y el desempeño de las agencias gubernamentales de asistencia técnica.

Esto permitió que las cooperativas se organizaran y adquirieran el tamaño de grandes empresas, configurando el sector agroindustrial, iniciando un proceso de incorporación de pequeñas cooperativas, concentrando el sector, y se liquidaron otras cooperativas por no incorporarse al proceso de concentración empresarial.

Esse modo de trabalhar lhe é apresentado como uma "saida" à falta de alternativas que lhe garanta a sua sobrevivência material. De certo modo, a induçāo acaba por despertar no trabalhador a curiosidade de "saber como é" e a possibilidade de "fazer diferente" passa a povoar a sua mente, o seu sonho, mas não o seu bolso. O "fazer diferente" exerce uma forte atração como nossos resultados evidenciaram. As empresas coletivas solidárias são prisioneiras de um conflito multifacetado cujos lados são: o desejo de <fazer diferente> (que não é verdadeiramente de cada um, mas thes foi induzido pela politica pública), ou seja, organiza-se coletivamente movido por valores morais, pela solidariedade e motivaçôes coletivas, mas tais empresas são permanentemente <desafiadas > pelas restriçóes e dificuldades econômicas." (CARLEIAL \& PAULISTA, 2008, p. 34). Moishe POSTONE (2014, p. 186) también establece que las formas de regulación laboral son formas de dominación indirecta, mientras que la esclavitud es una forma de dominación de la mano derecha. En el mismo sentido, está Farid EDI (2007) que cuestiona si la Economía Solidaria conduce a la ciudadanía o es una reproducción histórica del bienestar. 
Existe un movimiento de cooptación de las antiguas Empresas Solidarias, especialmente en el sur del país, para que adopten prácticas de organización y gestión capitalistas (ICAZA, 2004, p. 43) como condición de acceso al crédito oficial e incentivo fiscal programas. En el contexto económico de la época, la transformación era una cuestión de supervivencia de las empresas.

Estos problemas de concentración, que afectaron a miles de pequeños productores asociados o cooperativistas y que también perjudicaron a los trabajadores rurales, son responsables de la imagen negativa del cooperativismo creada ańos después, opinión pública que persiste hasta el día de hoy en la población. Esto se vio reforzado por el total distanciamiento de la OCB de las luchas por la redemocratización del país en la década de los 80 y su alienación en relación a los procesos de reconstrucción de las organizaciones sindicales de trabajadores, pequeños empresarios y agricultores familiares. Por si esto fuera poco, con el fin del régimen dictatorial y el retorno de la libertad sindical, la OCB realizó una campaña incansable para evitar cualquier cambio en la legislación del sector cooperativo, especialmente la remoción de la condición de representante exclusivo de el sistema cooperativo nacional.

Sin embargo, especialmente con la Constitución Federal de 1988 se produjo un nuevo ordenamiento social e institucional, creando normas constitucionales contrarias a la legislación cooperativa vigente, como el art. 5\% XVIII, que garantiza la libertad sindical en el país, quedando prohibida la injerencia del Estado en el sector.

A pesar de los cambios constitucionales y económicos que han impactado al cooperativismo desde entonces, la principal ley de cooperativas $(5.764 / 71)$ se ha mantenido vigente por más de cuarenta y cinco años. En la década de 1990 se presentaron varias propuestas legislativas para reemplazar la ley 5.764/71, pero ninguna ha sido aprobada hasta el momento, con pequeńas excepciones que amplían o corrigen el sistema cooperativo con la ley 9.867/99 (Cooperativas Sociales), Medida Provisional 2.168 -40 (crea Sescoop - Servicio Nacional de Cooperativas de Aprendizaje), la ley complementaria 130/09 (Cooperativas de Crédito), la ley 12.690/12 (Cooperativas Laborales), así como el Código Civil de 2002 que modifica temas de pequeñas empresas.

El punto central que impide la reforma de la legislación cooperativa proviene del tema de la representación única del movimiento. Por un lado, la Constitución Federal garantizó la libre asociación, resultando en la recepción no constitucional del sistema único de representación de la $\mathrm{OCB}$, la ausencia de un reglamento legal impide la consolidación del cooperativismo en el país, manteniendo la estructura institucional previa a la Constitución de 1988 y reproduciendo las correlaciones de fuerzas de los años 70 y 80 . 
La actuación de la OCB y sus entidades estatales (OCE) sigue siendo, en gran medida, una entidad paraestatal, actuando como un poder delegado y ejerciendo una función pública. Un ejemplo de esto es el registro obligatorio de nuevas cooperativas en la OCESP (Organización de Cooperativas del Estado de São Paulo) antes de la solicitud de registro de la empresa en la Junta Estatal de Comercio, de conformidad con el art. 3, $\$ 3$ de la Ley del Estado 12.226/06 y, la Deliberación 12/12 de JUCESP.

Esto explica en parte el crecimiento en el ritmo de establecimiento formal de cooperativas en Brasil. El aumento se produce principalmente después de 1994 como resultado de la estabilidad monetaria. Entre 1990 y $1995^{7}$ el promedio de cooperativas establecidas fue de seiscientas quince por año, con el cierre promedio de actividades de solo dieciocho. Sorprendentemente, entre 1996 y 2001, el promedio de registros saltó a dos mil ciento noventa y tres por año, y la tasa de cierre se elevó a cincuenta y ocho.

Sin embargo, a pesar de que hubo un movimiento conservador en la expansión del cooperativismo brasileño (antes de la década de 1990), que condujo a una política de aumento de las desigualdades regionales, no se puede decir que existiera un modelo cooperativo único. Como afirma João Elmo SCHNEIDER (1981, p. 19), a "dinâmica do modelo de acumulaçáo de capital vigente no país, cuja característica fundamental é o desenvolvimento desigual da sociedade brasileira" o aún, como ejemplificas RIOS (1987, p. 18):

Existe um cooperativismo de elites e um cooperativismo dos pés-no-chão; um cooperativismo legalizado, letrado e financiado e um cooperativismo 'informal', 'sem lei e sem documento', não financiado e mesmo reprimido. O cooperativismo não está pois 'imunè à divisâo da sociedade em classes.

Si bien el "modelo oficial" del cooperativismo brasileño se construye de arriba hacia abajo, desconectado de las necesidades de las bases sociales, el concepto central del cooperativismo está regresando y ampliando el papel de la inclusión social.

7. El ramo que más se incrementó fue el de las cooperativas de trabajo como consecuencia de la ley 8.949/94, que modifica el art. 442, Párrafo Único de la CLT para declarar la inexistencia de relación laboral entre cooperativa y cooperativista, incluso en las cooperativas de trabajo. Esta alteración del CLT (que reproducía el art. 90 de la Ley 5.764/71) abrió el camino a la subcontratación a través de cooperativas de trabajo, muchas de ellas prestando servicios a los poderes públicos y empresas estatales. 


\subsection{Cooperativas agrícolas en Brasil}

Dado el papel protagónico de las cooperativas agrícolas en Brasil, especialmente en los años 70 con la creación de la cooperativa actual, es necesario analizar las características de las cooperativas rurales, especialmente dado su papel en la concentración de la tierra por un lado y la supervivencia de los pequeños. empresas agrícolas familiares de otro.

Lo que se observa en la evolución del cooperativismo agrícola en Brasil es que se inicia en las áreas rurales y a partir de la década del 40 del siglo pasado comienza a migrar al medio urbano, dejando esta rama original del cooperativismo, que dio origen a todo el movimiento cooperativo y también la Economía Solidaria. Por ello, merece un subcapítulo específicamente dirigido al cooperativismo agrícola.

El uso del sistema cooperativo en el ámbito rural, aunque es el fundador del cooperativismo en Brasil, solo cobró fuerza en la segunda mitad del siglo pasado, impulsado especialmente por la propuesta de trabajo comunitario y fincas del Partido Comunista Brasileño (PCB) y por sectores progresistas del la Iglesia Católica.

El origen, sin embargo, es anterior, comenzando especialmente con la inmigración europea en el siglo XIX y la introducción en el país de las asociaciones agrarias, que se da en un contexto plural a la vista de la colonización previa de portugueses y africanos. Las etapas de la inmigración moldearon la cultura campesina del país, estructurada en el mestizaje racial y, en la confluencia y reinvención cultural resultante de las condiciones climáticas, el suelo y el aislamiento continental de Brasil.

Organizadas en todas las zonas ocupadas del país, las asociaciones cobraron mayor protagonismo en las regiones donde existían colonias europeas formadas por inmigrantes llegados tras el auge del capitalismo como sistema económico en Europa en el siglo XIX (hecho que excluye a los portugueses). La inmigración precapitalista anterior no tenía experiencia práctica ni teórica de organización de asociaciones y cooperativas, ni siquiera colonias cooperativas o falansterios, defendidos por los socialistas utópicos anglo-franceses y luego por los anarquistas italo-españoles.

Las asociaciones traídas desde Europa no fueron meramente productivas, destacando, como ejemplo, en las colonias alemanas (urbanas y rurales) ubicadas en el sur del país, asociaciones culturales, deportivas y económicas, conformando una marańa de iniciativas comunitarias que perduran hasta el día de hoy. En los primeros ańos del siglo pasado se catalogaron en Rio Grande do Sul aproximadamente trescientas cincuenta asociaciones de composición alemana (RAMBO, 1988, p. 46). En el mismo período, los colonos italianos se destacaron por la creación de sociedades de ayuda mutua, asociaciones que se perdieron en la década de 1930. 
En la década de 1930 (inicio del primer gobierno de Getúlio Vargas), el cooperativismo se convirtió en un instrumento de política agrícola, atrajo la atención del Estado y dejó de ser visto como una amenaza anarquista, como lo demuestra el surgimiento de las primeras leyes que regulan el cooperativismo agrícola. Veinte ańos después, a fines de la década de 1950, para modernizar la agricultura (especialmente el trigo y la soja), el cooperativismo se convirtió en la principal herramienta del desarrollo rural, permitiendo la inserción de Brasil en el mercado internacional (estrategia que se repetirá en la legislación de 1971) mediante la formación de empresas comerciales de exportación.

Las cooperativas agrarias, por un lado, se convirtieron en el nexo entre la agricultura familiar y los modernos sistemas de producción agraria y, por otro, terminaron contribuyendo al surgimiento de un nuevo perfil empresarial en el campo, vinculando productores, instituciones públicas de desarrollo y consumidores. mercado, bifurcación que luego fue responsable de la separación entre cooperativismo y agricultura familiar.

El carácter emprendedor se deriva del estímulo estatal ${ }^{8}$, lo cual, ligado a las fracciones de la burguesía urbana y rural. En este espíritu capitalista, los agricultores familiares se organizan "funcionalmente" a través de cooperativas comerciales, lo que en última instancia conduce a la incorporación de pequeñas cooperativas tradicionales por parte de las cooperativas comerciales. Este proceso se caracteriza por la centralización y concentración que llevó a la extinción de cooperativas, asociaciones y agricultores que no adoptaron estructuras empresariales. Estas nuevas cooperativas se vincularon umbilicalmente con políticas oficiales (especialmente crédito) y, con los mercados consumidores internacionales, convirtiéndose en uno de acceso al crédito oficial subsidiado y la incorporación de nuevas tecnologías productivas.

Durante el proceso de concentración organizacional (especialmente después de la Ley 5.764/71), crecimiento económico y expansión territorial de la frontera agrícola (hacia el Medio Oeste y Amazonas), el cooperativismo rural acumuló contradicciones y crisis, presentando fallas y límites, necesitando cambiar sus estrategias de ac-

8. Un ejemplo importante de la participación (ideológica) de los gobiernos militares en el fomento de determinadas cooperativas agrícolas es la CAC - Cooperativa Agrícola de Cotia. La base de la regulación social del movimiento campesino a través de la organización de las cooperativas fue estimulada por los militares que alentaron, de todas las formas posibles, la expansión de las actividades de algunas cooperativas como CAC a zonas fronterizas como la Amazonia y el Medio Oeste. Para Dráuzio Leme PADILHA (1989, p. 241), historiador de CAC, esta cooperativa para expandirse al Medio Oeste recibió el apoyo del INCRA - que se encargó de expropiar tierras para distribuirlas a los miembros de la cooperativa; BDMG, que financió la infraestructura y los cultivos; EMATER: proporcionó asistencia técnica a los miembros; BANCO DO BRASIL - con financiamiento rural, y MINAS-CAIXA, que financió las obras de infraestructura para la instalación de la cooperativa. 
ción. conduciendo al surgimiento (en realidad, una reanudación) de una nueva línea de acción. El retorno al principio de autogestión, encontrado a finales de los años 80, marca el inicio de una nueva etapa, orientada a impulsar las actividades económicas, invertir en nuevas tecnologías y estrategias de acción política eficaces. A partir de esto, el cooperativismo brasileño se dividió a fines del siglo XX, teniendo actualmente dos formas de organización y representación ideológicamente diferentes, a saber: el cooperativismo "tradicional", con sesgo empresarial, y el cooperativismo "popular" con sesgo de resistencia que permea el debate (y dualidad) de toda la discusión hoy, no solo en el sector rural. 
(pp. 285-303)

\section{Referencias}

BULGARELLI, W. (1995): Questóes atuais de direito empresarial. (1 $1^{\mathrm{a}}$ ed.). Malheiros. CARLEIAL, L. \& PAULISTA, A. (2008): Economia Solidária: Utopia Transformadora ou Política de Controle Social?. Estudos de Direito Cooperativo e Cidadania, 2, 9-40.

CUNHA, G. \& SANTOS, A.M. (2011): Economia Solidária e pesquisa em ciências sociais: Desafios epistemológicos e metodológicos. En P. HESPANHA \& A. M. SANTOS (Orgs.), Economia Solidária: Questões Teóricas e Epistemológicas (pp. 1556). Almedina.

EDI, F. (2007): Descentralização do estado, economia solidária e políticas públicas: Construção da cidadania ou reproduçáo histórica do assistencialismo?. $O R G$ \& DEMO, 8(2), 47-66. https://doi.org/10.36311/1519-0110.2007.v8n1/2.382

GUIMARÃES, A.Q. (2011): Iniciativas para a promoção de emprego e renda: políticas públicas, economia solidária e desenvolvimento local. Ensaios FEE, 32(2), 313-338.

ICAZA, A. M. S. \& ASSEBURG, H.B. (2004): Autogestão e viabilidade em cooperativas de calçado no Rio Grande do Sul. En: L. I. GAIGER (Org.), Sentidos e experiências da economia solidária no Brasil (pp. 55-79), Editora da UFRGS.

MAURER JÚNIOR, T. H. (1966): O Cooperativismo - uma economia humana. (1 ${ }^{\mathrm{a}}$ ed.). Metodista.

MELLO NETO, C. (1998): O anarquismo experimental de Giovanni Rossi. De Poggio al Mare à Colônia Cecília. (2a ed.). Editora UEPG (Universidade Estadual de Ponta Grossa).

PADILHA, D.L. (1989): CAC-Cooperativismo que deu Certo. Cooperativa Agrícola de Cotia.

PINHO, D. B. (1996): Manual de Cooperativismo. (Vol. III). Conselho Nacional de Desenvolvimento Científico e Tecnológico (CNPq).

POSTONE, M. (2014): Tempo, Trabalho e Dominação Social. (1'a ed.). Boitempo.

RAMBO, A. B. (1988): O associativismo teuto-brasileiro e os primórdios do cooperativismo no Brasil. Eduni.

RIOS, G. (1987): O que é cooperativismo?. Brasiliense.

SCHNEIDER, J.E. (1981): O cooperativismo agrícola na dinâmica social no desenvolvimento periférico dependente: $\mathrm{O}$ caso brasileiro. En M.R. LOUREIRO (Org.), Cooperativas agrícolas e capitalismo no Brasil (pp. 11-40), Cortez. 
SOCHODOLAK, H. \& MANEIRA, R. (2011): Os faxinais de Irati na década de 1940: a força de uma cultura tradicional. En Anais do XI Congresso Luso-afro-brasileiro de Ciências Sociais (pp. 1-16). Salvador.

VEIGA, S. M. \& FONSECA, I. (2001): Cooperativismo: uma revolução pacífica em ação. DP\&A.

WEBER, M. (2004): Economia e Sociedade. (4a ed., Vol. 1). Editora UnB (Universidade de Brasília).

ZECCA CASTEL, R. (2008): Il positivismo anarchico di Giovanni Rossi. L'esperimento di una comune libertaria nel Brasile della fine del XIX secolo. Università degli Studi di Milano, Tesi di Laurea. 